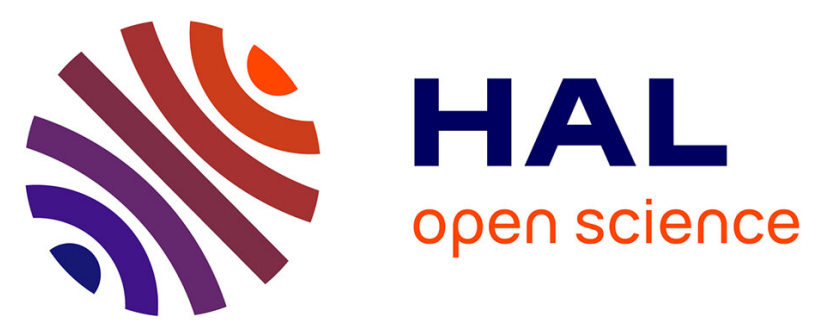

\title{
Self-Assembly of Hydrophobin Protein Rodlets Studied with Atomic Force Spectroscopy in Dynamic Mode
}

S. Houmadi, R. Rodriguez, S. Longobardi, P. Giardina, Marie-Claude Fauré, M. Giocondo, Emmanuelle Lacaze

\section{- To cite this version:}

S. Houmadi, R. Rodriguez, S. Longobardi, P. Giardina, Marie-Claude Fauré, et al.. Self-Assembly of Hydrophobin Protein Rodlets Studied with Atomic Force Spectroscopy in Dynamic Mode. Langmuir, 2012, 28, pp.2551. 10.1021/la2028093 . hal-00777900

\section{HAL Id: hal-00777900 https://hal.science/hal-00777900}

Submitted on 18 Jan 2013

HAL is a multi-disciplinary open access archive for the deposit and dissemination of scientific research documents, whether they are published or not. The documents may come from teaching and research institutions in France or abroad, or from public or private research centers.
L'archive ouverte pluridisciplinaire HAL, est destinée au dépôt et à la diffusion de documents scientifiques de niveau recherche, publiés ou non, émanant des établissements d'enseignement et de recherche français ou étrangers, des laboratoires publics ou privés. 


\section{Self-assembly of hydrophobin protein rodlets studied with atomic force spectroscopy in dynamic mode}

S. Houmadi ${ }^{a, b}$, Raul D. Rodriguez ${ }^{a, c}$, S. Longobardi ${ }^{d}$, P. Giardina ${ }^{d}$, M. C. Fauré ${ }^{a}$, M. Giocondo ${ }^{b}$ and E. Lacaze $^{a}$

${ }^{a}$ Institut des Nano-Sciences de Paris (INSP), UMR-CNRS 7588, Université Pierre et Marie Curie-Paris 6, 4 place Jussieu 75005 PARIS, France.

${ }^{b}$ CNR-IPCF - UOS di Cosenza. c/o Dipartimento di Fisica, Università della Calabria, 87036 Rende, Italy.

${ }^{c}$ Institute of Physics, Chemnitz University of Technology, 09107 Chemnitz, Germany

${ }^{d}$ Dipartimento di Chimica Organica e Biochimica, Università di Napoli Federico II, Via Cintia 4, 80126 Napoli, Italy.

houmadi3@yahoo.fr, raulmet@gmail.com, sara.longobardi1983@libero.it, giardina@unina.it, marie-claude.faure@univ-paris5.fr, michele.giocondo@cnr.it,Emmanuelle.Lacaze@insp.jussieu.fr

Corresponding author. E-mail: Emmanuelle.Lacaze@insp.jussieu.fr Tel: +33144274654. Fax: $+33143542878$

ABSTRACT. 
We have investigated the self-assembling properties of the class I hydrophobin Vmh2 isolated from the fungus Pleurotus ostreatus. Five different hydrophobin self aggregate samples including monolayers, bilayers and rodlets have been prepared by Langmuir technique and studied at the nanoscale. Local wettability and visco-elasticity of the different hydrophobins samples were obtained from Atomic Force Spectroscopy experiments in dynamic mode performed at different, controlled relative humidity (RH) values. It was found that hydrophobins assembled either in rodlets or in bilayer films, display similar hydropathicity and viscoelasticity in contrast to the case of monolayers, whose hydropathicity and viscoelasticity depend on the adopted preparation method (Langmuir-Blodgett or Langmuir-Schaeffer). The comparison with monolayers properties evidences a rearrangement of the bilayers adsorbed onto solid substrates. It is shown that this rearrangement leads to the formation of a stable hydrophobic film, and that the rodlets structure consists in fragments of restructured proteins bilayers. Our results support the hypothesis that the observed variations in the viscoelastic properties could be ascribed to the localization of the large flexible loop, typical of Class I hydrophobins which appears free at the air interface for LB monolayers but not for the other samples. These findings should now serve future developments and applications of hydrophobin films beyond the archetypal monolayer.

\section{KEYWORDS.}

Langmuir films, Hydrophobin proteins, Rodlets, AFM, AFS, Capillarity, Viscoelasticity.

\section{Introduction}

Hydrophobins are amphiphilic proteins produced exclusively by fungi ${ }^{1}$. They form an amphipathic layer which self-assembles at hydrophobic and hydrophilic surfaces and are the most surface active proteins known $^{2,3}$. It has been shown that hydrophobicity of surfaces can be reversed using hydrophobin films ${ }^{2,}$ 4-8. Recently, research on these proteins has focused on utilizing their physical properties for biotechnological applications ${ }^{3,9,10}$. In biosensor design hydrophobins allow the subsequent attachment of cells or proteins ${ }^{6,11}$. Hydrophobins are divided into two groups, class I and class II hydrophobins. It 
is well-known that at the air-water interface, molecules of class I self-assemble to form anisotropic aggregates, the so-called rodlets ${ }^{12}$. Rodlets may also be formed in vitro on both hydrophobic and hydrophilic surfaces ${ }^{13}$. They are extremely stable and can be dissolved only in pure trifluoro acetic acid. This property makes them useful for application in nanotechnology ${ }^{14}$. The formation of the rodlet-like film is accompanied by beta-sheet structure formation ${ }^{15}$. Models proposed to account for the formation of rodlets interpret them as corresponding to a monolayer ${ }^{13,16,17}$. However, in a previous paper, we have demonstrated the coexistence of rodlets and monolayer ${ }^{18}$ in the same film, suggesting the hypothesis of a hydrophilic double-layer structure for the rodlets possibly stabilized by the presence of the surrounding monolayer. Our aim in this work is to advance in the understanding of the structural conformation taken by self-assembled class I hydrophobins that are purified from the basidiomycete fungus Pleurotus ostreatus and identified as $\mathrm{Vmh} 2^{19}$. We have investigated physical properties of five different structures: two monolayer films prepared by Langmuir-Schaefer (LS) or Langmuir-Blodgett (LB) methods, two bilayer films obtained by a combination of both methods, and rodlets using Atomic

Force Microscopy (AFM) and Atomic Force Spectroscopy (AFS) in dynamic mode. The relevance of this latter technique is here evidenced by providing new insights about wetting properties and viscoelasticity of films and aggregates, leading to new assumptions concerning structure of hydrophobin films as well as the molecular rearrangement during films and rodlets formation.

Materials and Methods.

\section{Hydrophobin Purification.}

The Vmh2 hydrophobin protein was purified from the fungus Pleurotus ostreatus as described in the reference [19]. Briefly, mycelia were grown at $28^{\circ} \mathrm{C}$ in static cultures in potato dextrose $(24 \mathrm{~g} / \mathrm{L})$ broth with $0.5 \%$ yeast extract. Hydrophobins released into the medium were aggregated by air bubbling. Foam was then collected by centrifugation at $4000 \mathrm{x}$ g. The precipitate was freeze dried and treated with trifluoroacetic acid (TFA) . The sample was then dried, dissolved in $60 \%$ ethanol and centrifuged.

\section{LB/LS films.}


The Langmuir films were prepared both in a Nima Tech 632D1D2 LB system trough and a home-built trough using ultrapure millipore water, at $5.5 \mathrm{pH}$ and $T=18.0 \pm 0.5{ }^{\circ} \mathrm{C}$ for the subphase ${ }^{18}$. Once Vmh2 deposited at the air/water interface, the monolayer was compressed up to the transfer pressure of 36 $\mathrm{mN} / \mathrm{m}^{18}$. Before the film transfer, we compressed the monolayer at the air-water interface five times to obtain coexistence between rodlets and monolayer but only once to obtain an homogeneous monolayer. The LB monolayers were transferred at constant pressure of $36 \mathrm{mN} / \mathrm{m}$ onto hydrophilic $\mathrm{SiO}_{2}$ substrates, by vertical lifting, at a rate of $10 \mathrm{~mm} / \mathrm{min}$. Transfer was similar for coexisting rodlets and monolayer. Silanized silicon wafers were used for the LS transfer that allows by horizontal lifting method to deposit films with the hydrophilic side in contact with air (LS film). $\mathrm{SiO}_{2}$ wafers were silanized using octadecyltriethoxysilane (OTE), according to the procedure described by Malham et al. ${ }^{20}$. In order to obtain a bilayer with hydrophilic surface in contact with air, a monolayer (compressed once) was deposited using LS technique onto an already-prepared Vmh2 LB film (this bilayer is named hereinafter LB+LS). Conversely, in order to obtain a bilayer with hydrophobic surface in contact with air, a monolayer (compressed once) was deposited using vertical lifting onto Vmh2 LS film (this bilayer is named hereinafter LS+LB).

\section{Contact Angle Measurements.}

Contact angles of water on samples were measured on a home-built instrument. Each surface, placed on a horizontal holder, was brought into contact with a droplet of ultrapure water hanging from the vertical needle of a $10 \mu \mathrm{L}$ microsyringe. Video acquisition was performed using a CCD camera and a long working-distance objective on 3 to 5 different locations.

\section{Atomic Force Microscopy (AFM), Spectroscopy and Imaging.}

AFM measurements were obtained in tapping mode using two apparata from Veeco Instruments: Nanoscope Dimension 3100 and MultiMode. Oxidized silicon cantilevers purchased from NanoSensors were used with tips radius of the order of $10 \mathrm{~nm}$. The AFM measurements were performed in ambient 
conditions (i.e. $40 \% \mathrm{RH}$ ) as well as in controlled RH. Humidity control was achieved by placing the microscope inside a glass chamber and using a home-made humidifier to vary the ratio of dry/wet nitrogen flowing into the chamber. $\mathrm{RH}$ was measured with a precision of $\pm 0.5 \% \mathrm{RH}$. To avoid local gradients of water vapor, the system was left to stabilize for at least half an hour before each measurement. Prior to the AFS measurements (with no sample beneath) the AFM cantilever was excited at its resonance frequency (around $150 \mathrm{kHz}$ ) and the phase-distance curves on the sample were conducted at the same frequency. To ensure the appropriate comparison between AFS data, we checked that the AFM tip underwent no changes during the entire experiment: AFM tips were tested against a $\mathrm{SiO}_{2}$ surface used as a reference. Phase distance curves on the reference $\mathrm{SiO}_{2}$ surface were compared before and after each measurement performed on the Vmh2 film; phase data that showed differences on the reference $\mathrm{SiO}_{2}$ surface were taken as due to a modified (i.e. : blunted) tip and discarded.

\section{Results and Discussion}

Using Langmuir method, we formed hydrophobin monolayers at the air/water interface, compressed only once, which were then transferred by LB technique onto a hydrophilic $\mathrm{SiO}_{2}$ substrate for one film (LB film) and by LS technique on a hydrophobic silanized $\mathrm{SiO}_{2}$ substrate for the other film (LS film). In both LB and LS depositions, Vmh2 molecules formed homogenous films as shown in Fig. 1A and 1B from AFM imaging. The similarity in topography between both films suggests that LS transfer, although less accurate than the LB one, does not disrupt the hydrophobin interfacial film more than LB transfer, as already reported for HGFI class I hydrophobin ${ }^{21}$. From detailed AFM analysis, same low roughness RMS of $0.25 \mathrm{~nm}$ has been found. Possibly due to some inhomogeneity of the substrate, rarely, holes could be found in the LB and LS monolayers, allowing for the thickness measurement by AFM. Same value of $2.5 \pm 0.4 \mathrm{~nm}$ has been found for LB and LS monolayers (fig. 1). 2.5nm corresponds to the hydrophobin size in solution ${ }^{3,}$ it is thus associated with monolayer films. On the other hand, as already reported ${ }^{18,21}$, films prepared by repeating compression-expansion cycles at the airwater interface and then deposited on $\mathrm{SiO}_{2}$ substrates showed rod-like aggregates characteristic of class 
I hydrophobins (see Fig. 1C taken from [18]). These rodlets appear to coexist with the hydrophobin monolayer, and they appear $2.5 \mathrm{~nm}$ higher than the surrounding monolayer. This shows that rodlet's height is of $5 \mathrm{~nm}$ which may imply two different hypotheses about their structure: 1) rodlets are made of a monolayer of proteins that have undergone strong conformational changes in order to explain the large deviation from the size the protein has in solution, but this scenario is unlikely to occur due to the tight binding cores of hydrophobins. 2) rodlets are made out of two coupled monolayers with two possible structures: either bilayers are disconnected from the surrounding monolayer, or a monolayer patch is directly adsorbed onto the underlying monolayer. During the monolayer compression in the Langmuir through, a part of the hydrophobin molecules could be expelled from the monolayer and they would form stripes predominantly parallel to the barrier.

LB and LS layers homogeneity allows to investigate the wettability by contact angle measurements. Due to the LB method transfer on hydrophilic surface, Vmh2 molecules are attached to the hydrophilic $\mathrm{SiO}_{2}$ by their hydrophilic parts, while the hydrophobic regions of the protein remain exposed to the air and the contrary is consistently expected for LS films. LB film exhibits a contact angle of $81^{\circ}$ which means that it is indeed more hydrophobic than hydrophobin LS film that displays a contact angle of $43^{\circ}$. The contact angle value obtained for LS film is consistent with those reported in Ref. [2] for class I hydrophobins on Teflon (from $36^{\circ}$ to $63^{\circ}$ ). The dispersion in the contact angle values ${ }^{2,6}$ appears related to the hydrophobin exact nature but also to the dispersion of preparation method. In this context, it is worthwhile to notice that the LB and LS techniques allow to obtain hydrophobin layers with not only well defined thickness, but also well defined molecular orientation if it is not modified by the transfer onto substrate.

\section{Surface Measurements at the Nanoscale.}

Modeling Phase Vs. Distance Curves. AFM in tapping mode works with a tip attached to an oscillating cantilever, at a frequency close to its resonance frequency $\omega_{0}$. For AFM imaging, the oscillation amplitude is fixed in order to record the surface topography. In AFS, the variations of the 
oscillation amplitude, $A$ and phase, $\Phi$, are recorded as a function of the average tip-sample distance $\mathrm{d}_{0}$. They reflect the perturbation of the cantilever dynamics by the interaction between the tip and the sample. The AFM cantilever can be modeled as a forced harmonic oscillator with $\mathrm{k}_{\mathrm{c}}$ the stiffness and $\mathrm{Q}$ the quality factor associated with damping in air. In presence of tip-sample interactions $F_{t s}(d)$, the equation of motion is given by :

$$
\frac{k_{c}}{\omega_{0}^{2}} \frac{d^{2} z(t)}{d t^{2}}+\frac{k_{c}}{\omega_{0} Q} \frac{d z(t)}{d t}+k_{c} z(t)=F_{0} \cos (\omega t)+F_{t s}\left(d_{0}+z(t)\right)
$$

where $z(t)$ is the oscillation of the tip. Notice that $d_{o}$ in simulations and the vertical displacement in experimental curves are equivalent except for an arbitrary offset value. Contact occurs when $d_{o}-A=a_{o}$ with $a_{o}$ the tip-sample distance at which contact interactions start taking place. The tip-sample interaction $\mathrm{F}_{\mathrm{ts}}(\mathrm{d})$ may be defined by a combination of van der Waals forces (vdW) and DerjaguinMuller-Toporov contact mechanics (DMT). The presence of viscoelasticity is taken into account through a damping within the sample:

$$
\left\{\begin{array}{l}
d_{0}+z(t) \geq a_{0}, F_{t s}=-\frac{H R}{6\left(d_{0}+z(t)\right)^{2}} \\
d_{0}+z(t) \leq a_{0}, F_{t s}=\frac{4}{3} E\left(R\left(d_{0}+z(t)\right)^{3}\right)^{1 / 2}-\frac{H R}{6 a_{0}^{2}}-\frac{k_{c}}{\omega_{0} Q_{d i s}} \frac{d z(t)}{d t}
\end{array}\right.
$$

In this equation, $\mathrm{H}$ represents the Hamaker constant of the tip-sample system, $\mathrm{E}$ is the reduced Young modulus, and $\mathrm{R}$ is the tip radius which is herein assumed to be spherical. $\mathrm{Q}_{\mathrm{dis}}$ is the quality factor associated with damping of the tip within the sample. The numerical integration of the equation of motion was performed by using a fourth order Runge-Kutta algorithm ${ }^{22}$. The phase values calculated are plotted as a function of $d_{o}$ in Fig. 2. The transition between regime dominated by attractive long range forces and repulsive ones has a clear signature in phase curves given by a jump from values larger than $90^{\circ}$ to values below $90^{\circ 23}$. When viscoelasticity is taken into account, this shift is modified, as shown on Fig. 2. Phase/distance curves can also be used to calculate the power dissipated by the tip-sample 
interaction. When the cantilever is excited at its resonance frequency, the power dissipated by the tip when it probes periodically the sample surface (i.e. energy dissipation per period) is obtained using the following equation ${ }^{24}$ :

$$
P_{\text {tip }}=\frac{1}{2} \frac{k_{c} A^{2} \omega_{0}}{Q}\left[\frac{A_{0}}{A} \sin \phi-1\right]
$$

where $A_{o}$ is the free amplitude of the cantilever. For conservative forces like vdW or DMT ones, the power dissipated by the tip is expected to be zero. The evaluation of the power dissipation thus allows to highlight non conservative interactions like viscoelastic ones.

Hydrophobicity investigation at the nanoscale. Wetting properties of our Vmh2 samples were further investigated at the nanoscale, using AFS. Changes in humidity have a role in adhesion forces between the AFM tip and the sample, due to the formation and growth of a water bridge between both surfaces ${ }^{25,}$ ${ }^{26}$. In Fig. 3A, phase versus distance curves performed on LS monolayer are presented for 4 RH values. At low RH (smaller than 30\%) the phase jump from the attractive regime to the repulsive one takes place at ca. $7 \mathrm{~nm}$ from the onset of tip-sample interactions (marked with " $\mathrm{A}$ " in the plot). When $\mathrm{RH}$ attains the critical value $34 \%$, the phase jump suddenly moves at ca. $10 \mathrm{~nm}$ from "A". This behavior is ascribed to the absence of meniscus between the tip and the sample at low RH: the water vapor does not condensate between tip and sample, and the tip-sample interaction is mainly dominated by attractive $\mathrm{vdW}$ and repulsive contact forces. When the RH attains $34 \%$ the formation of a water bridge between the tip and the LS film results in a shift of the jump toward the left; this is associated with the energy dissipation required to break the meniscus ${ }^{27}$. This kind of evolution was systematically observed on LS films for all the tips used with a threshold humidity only varying between $25 \% \mathrm{RH}$ and $38 \% \mathrm{RH}$. In contrast, the phase curve related to the LB films, evidences no variation in the position of the jump for humidity varying from 3\%RH to $79 \% \mathrm{RH}$ (Fig. 3B). This confirms that, at the local scale also, the LB film is more hydrophobic than the LS one, with no water nano-condensation between tip and surface taking place (table I). These results confirm also the ability of AFM in tapping mode to probe the 
wettability of surfaces at the nanoscale, which allows now to investigate locally the hydrophobicity of rodlets. Notice that this task would not have been possible to perform using contact angle measurements where wettability is averaged over a large sample area including rodlets and the LB film.

We have thus localized the AFM tip on rodlets (co-existing in the same sample with LB monolayer (see Fig. 1C)). On the top of the rodlets, we observe no changes in the Phase Vs. distance curves when the RH increases (Fig. 3C), similarly to the LB monolayer surrounding the rodlets. This behavior is contrary to what one would expect if we assume a bilayer thickness for the rodlets, with a hydrophilic exposed surface. We could thus expect that rodlets are formed by two hydrophobin layers on top of each other, interacting due to the presence of hydrophobic forces, independently of the hydrophilicity of the two layers facing each other. Hydrophobic forces indeed appeared dominant for hydrophobins of class $\mathrm{II}^{10}$, where attraction is dominated by hydrophobic interaction rather than by the electrostatic attraction. However in such a case we would expect a poor stability of rodlets on substrates at air, due to the absence of water which is not compatible with hydrophobic forces, contrary to our observations of extremely high stability of rodlets on silicon substrates. According to previously reported data ${ }^{15,16}$, conformational changes in some parts of the protein molecule could occur within rodlets, made of rearranged protein bilayer fragments (table I). It appeared thus interesting to study the local properties of Vmh2 bilayers by AFM/AFS for comparison with the rodlets properties. Using the Langmuir trough, we have prepared two kinds of bilayers, $\mathrm{LB}+\mathrm{LS}$ ones (LB below, LS on top, deposited on a $\mathrm{SiO}_{2}$ substrate) and $\mathrm{LS}+\mathrm{LB}$ ones (LS below, $\mathrm{LB}$ on top, deposited on a silanized $\mathrm{SiO}_{2}$ substrate). AFM images evidence the similarity in the topography of the two bilayers (Fig. 4). The roughness has increased with respect to monolayers, up to $0.8 \mathrm{~nm}$. Fig. 4 shows that, whatever the tip used is, wettability of LS+LB and LB+LS bilayers appears also similar and characterized by no formation of water meniscus between tip and surface, even at high RH. As expected, the LS+LB bilayer is hydrophobic, similarly to LB monolayer. Surprisingly, the LB+LS bilayer displays also a hydrophobic wetting behavior, while it is expected to present wetting properties comparable to LS films, i.e. hydrophilic. In order to account for this 
observation we conclude that structural changes must have occurred in the LB+LS bilayer, which is not a simple LS monolayer on top of a LB one (table I).

\begin{tabular}{|c|c|c|c|c|c|}
\hline \multirow{2}{*}{ Expected } & LB & LS & LS+LB & LB+LS & Rodlet \\
\hline Oydrophobic & Hydrophilic & Hydrophobic & Hydrophilic & - \\
\hline Observed & Hydrophobic & Hydrophilic & Hydrophobic & Hydrophobic & Hydrophobic \\
\hline
\end{tabular}

Table 1. Direct comparison of the surface hydropatic character at the nano scale of the different systems under investigation. With reference to a single monolayer floating on water, the hydrophobic side is represented in red whereas the hydrophilic one is represented in blue. The film arrangements shown in the schematics and the expected hydropathy behavior are those that would result if a rough amphiphilic molecule approximation is considered. The hydrophilic character is associated to the ability of a given system to give rise to nano condensation of water vapor between the afm tip and the sample surface for a critical value of the environmental Relative Humidity $(\mathrm{RH})$; the lower the critical RH value the higher the hydrophilic character. Conversely, when no water bridge forms whatever is the relative humidity, the surface is hydrophobic. Note that in the LB+LS case the observed hydrophobic behavior for the free surface is contrary to what one would expect for this assembly, and it could be the consequence of a protein reconfiguration inside the bilayer (see the text for the complete discussion).

Variations of Viscoelasticity at the Nanoscale. In order to validate this hypothesis, we have compared LS and LB monolayers first, then bilayers and rodlets, with the same tip under dry atmosphere to avoid 
any influence from capillarity. For LS and LB monolayers, systematically, as shown in Fig. 5A, the jump position for the LB monolayer is shifted with respect to the one of the LS monolayer by roughly 4 nm. Two hypotheses may explain such shift: either a particular attractive interaction exists between tip and LB monolayer; or viscoelasticity is different between both layers. In order to discriminate between the two hypotheses, we have used amplitude and phase-distance curves to obtain the power dissipated between tip and surface (equation 3). The dissipated power becomes different between LS and LB film for average distance between tip and surface smaller than the one associated with the jump between attractive and repulsive regimes (Fig. 5B). A different contact interaction thus occurs between the two films which can be attributed to differences in viscoelasticity, in agreement with numerical simulation results (see Fig. 2). The higher viscoelasticity corresponds to the LB film which presents larger power dissipation. The higher viscoelasticity for the LB monolayer compared to the LS one may be attributed to the asymmetry of the Vmh2 molecule. It is known that Class I hydrophobins are constituted by a tight binding core and a large flexible loop, markedly hydrophobic, between the Cys 3 and Cys $4^{13,17}$. The hydrophobic loop which is particularly flexible is also known to be much larger in the class I hydrophobins in comparison to the class $\mathrm{II}^{17}$. The higher viscoelasticity for the LB monolayer very likely can be related to the presence of this flexible loop. Due to its hydrophobicity the loop is expected to be in contact with air in the LB monolayer in order to minimize contact with the hydrophilic substrate; while in the LS monolayer the loop is expected to interact with the hydrophobic substrate, away from the air interface ${ }^{28}$. The flexibility of the loop at air may thus explain the difference in viscoelasticity observed for the two kinds of LB and LS hydrophobin monolayers. This result emphasizes the difference of structure between LB and LS monolayers, associated with the localization of the loop, which could not be detected with the AFM topography only.

Fig. 6A displays the comparison of phase distance curves, obtained with the same AFM tip, in dry conditions, related to LB monolayer and rodlets coexisting in the same sample. Systematically, the jump for the LB monolayer appears shifted towards smaller average tip-sample distances with respect to rodlets, with a shift of $3 \mathrm{~nm}$ for the tip used for the measurements reported on Fig. 6a. This shows that 
the dissipative term is less important in the rodlets than in LB monolayer, although both display similar hydrophobic character. In contrast to the LB films, in the rodlets the flexible part seems to be hidden from the air interface or rearranged in a more rigid structure. Similarly, Fig. 6B displays comparison between phase curves performed on top of both types of bilayer and of rodlets (still with the same tip). The position of the jump from the attractive regime to the repulsive one appears very similar for the two bilayers and for the rodlets with jump shifts differences not exceeding $1 \mathrm{~nm}$. This suggests that the three surfaces, $\mathrm{LB}+\mathrm{LS}, \mathrm{LS}+\mathrm{LB}$ and rodlets, have comparable viscoelasticity, lower than the LB one. The surface of LS+LB bilayer is consequently different from the one of LB monolayer deposited on hydrophilic rigid substrates. Unlike the LB film, the LS+LB bilayer does not expose the flexible parts to the air. Consequently, similarly to LB+LS bilayer, the hydrophobin molecules appear to reorganize in LS+LB bilayer, leading to an hydrophobic bilayer without the flexible part in contact with air. The properties of these films, as probed by AFM spectroscopy, are finally very close to the ones of LB+LS as well as to the ones of rodlets. These results, associated with the fact that rodlets present the same thickness than bilayers, led us to conclude that the three systems LB+LS, LS+LB and rodlets are equivalent. In other words, a stable configuration for interacting hydrophobins induced a rearrangement within LB+LS and LS+LB bilayers resulting in a structure similar to that of the rodlets. This rearrangement may have occurred during the deposition of the second layer and, more generally speaking, it would explain why homogeneous multilayers of hydrophobins are not possible to obtain using LB techniques ${ }^{29}$. Contrary to rodlets which always display an elongated form, LS+LB and LB+LS bilayers form uniform isotropic films. This difference may be associated with the formation of bilayers in two steps. The first LS or LB monolayer being isotropic, it may induce isotropy of the hydrophobin aggregates in bilayers. Rodlets, on the contrary, may show their characteristic preferential alignment as a direct result from the uniaxial compression in the Langmuir trough ${ }^{18,20}$. This implies in particular that rodlet units are already formed at the air/water interface before the film is transferred onto the substrate. It was already known that structural modifications occurred during spontaneous self-assembly of hydrophobins, at air/water interfaces or on substrates ${ }^{13,15,27,30}$. In this paper we now demonstrate that 
modifications also occur when bilayers are formed with Langmuir techniques and simply deposited onto hydrophilic or reversely onto hydrophobic substrates, independently of the nature of the substrate (hydrophilic $\mathrm{SiO}_{2}$ or hydrophobic silanized $\mathrm{SiO}_{2}$ substrates), leading to similar air interfaces. Similar modifications can occur when rodlets are formed. It is interesting to notice that in our conditions these modifications lead to formation of hydrophobic bilayers on top of a hydrophobic substrate, in contrast to other hydrophobin films obtained on hydrophobic Teflon, on which reversed rodlets (hydrophilic) are formed $^{31}$. On the other hand it is worthwhile to point out that in Ref 31 the hydropathy was evaluated through Water Contact Angle measurements on a macroscopic surface with a different protein (SC3). Rodlets formed by Vmh-2 hydrophobin are bilayers, hydrophobic, with a smaller visco-elasticity than

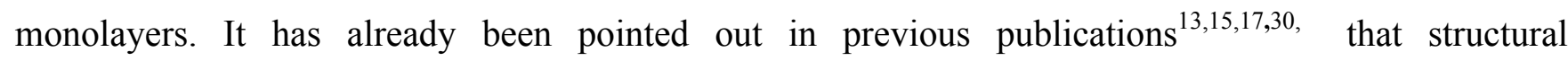
modification of hydrophobins occurred in the rodlets and that the loop, although not mandatory for rodlet formation, may be positioned between or around them and may affect their lateral packing. Our data can validate these hypotheses. The loop being not freely localized at the air/water interface, it may be possibly involved in interactions among molecules. Together with modification of hydrophobin structure within rodlets, future models should now take into account these new features for new modelizations of rodlets.

\section{Conclusion}

In summary, using force spectroscopy measurements in dynamic mode we have investigated Vmh2 LS and LB monolayers, rodlets and LS+LB, LB+LS bilayers at the nanoscale level. Concerning monolayers, we have confirmed the hydrophilicity of LS monolayer at the nanoscale by detecting the water meniscus formation between the AFM tip and the monolayer and demonstrated in contrast that no meniscus is formed between tip and LB monolayer. A higher viscoelasticity for LB monolayer has been evidenced with respect to the LS ones. This can be related to the presence of the loop, exposed to the air in the LB film, due to its hydrophobicity, but interacting with the substrate in the LS film. 
The fact that rodlets and bilayer films display similar hydropathicity and viscoelasticity demonstrates that bilayers are not formed by two "simple" hydrophobin monolayers (LS and LB monolayers) on top of each other but that rearrangement of hydrophobin molecules occured during the transfer of the second layer. It strongly suggests that rodlets are actually fragments of bilayers associated with structural modifications that lead to a common stable hydrophobic structure. The difference between hydrophobic bilayers and LB monolayer can be associated with loops no more freely located at the air interface in rodlets and bilayers and possibly involved in interactions between hydrophobins. It would be particularly interesting now to start simulations in order to build a model of interacting molecules in rodlets based on these experimental results.

ACKNOWLEDGMENTS. We thank Dr L. De Stefano for fruitful discussions, Dr I. B. Malham and Dr L. Bureau for substrate silanization and contact angle measurements and D. Limagne for technical support. RR and EL thank Dr. J. Jupille for valuable discussions.
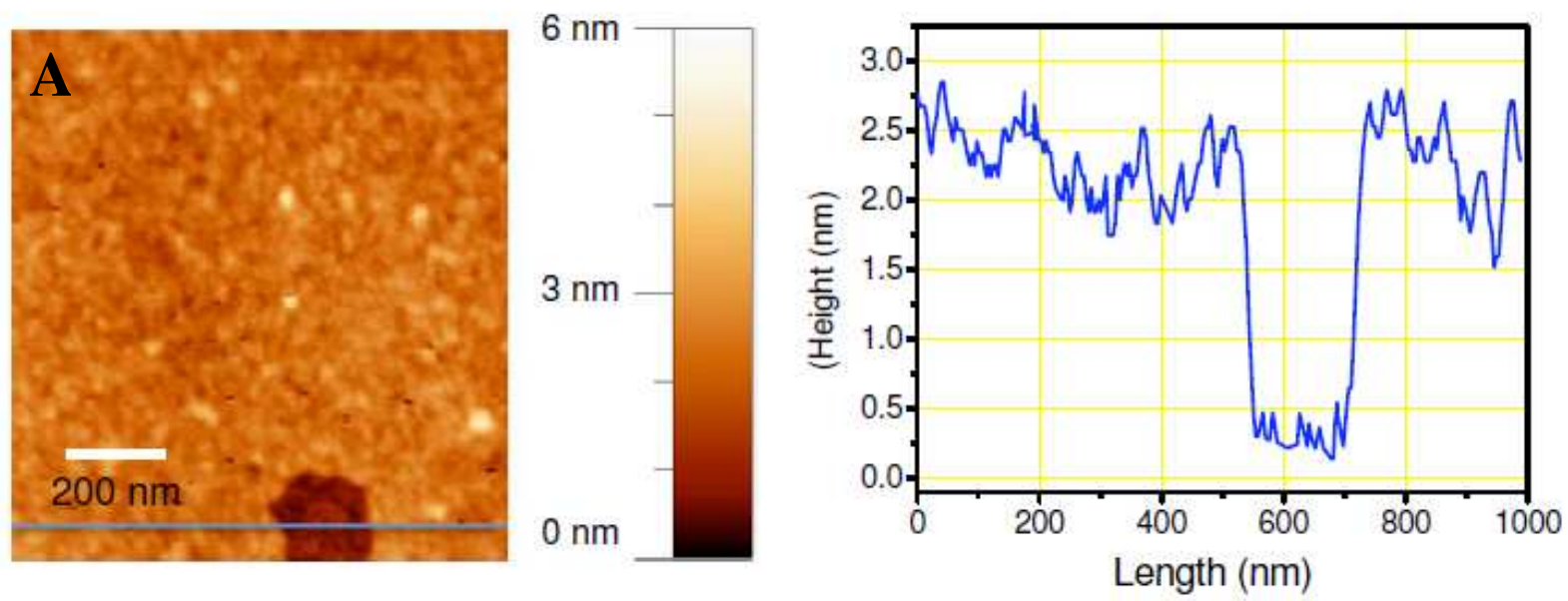

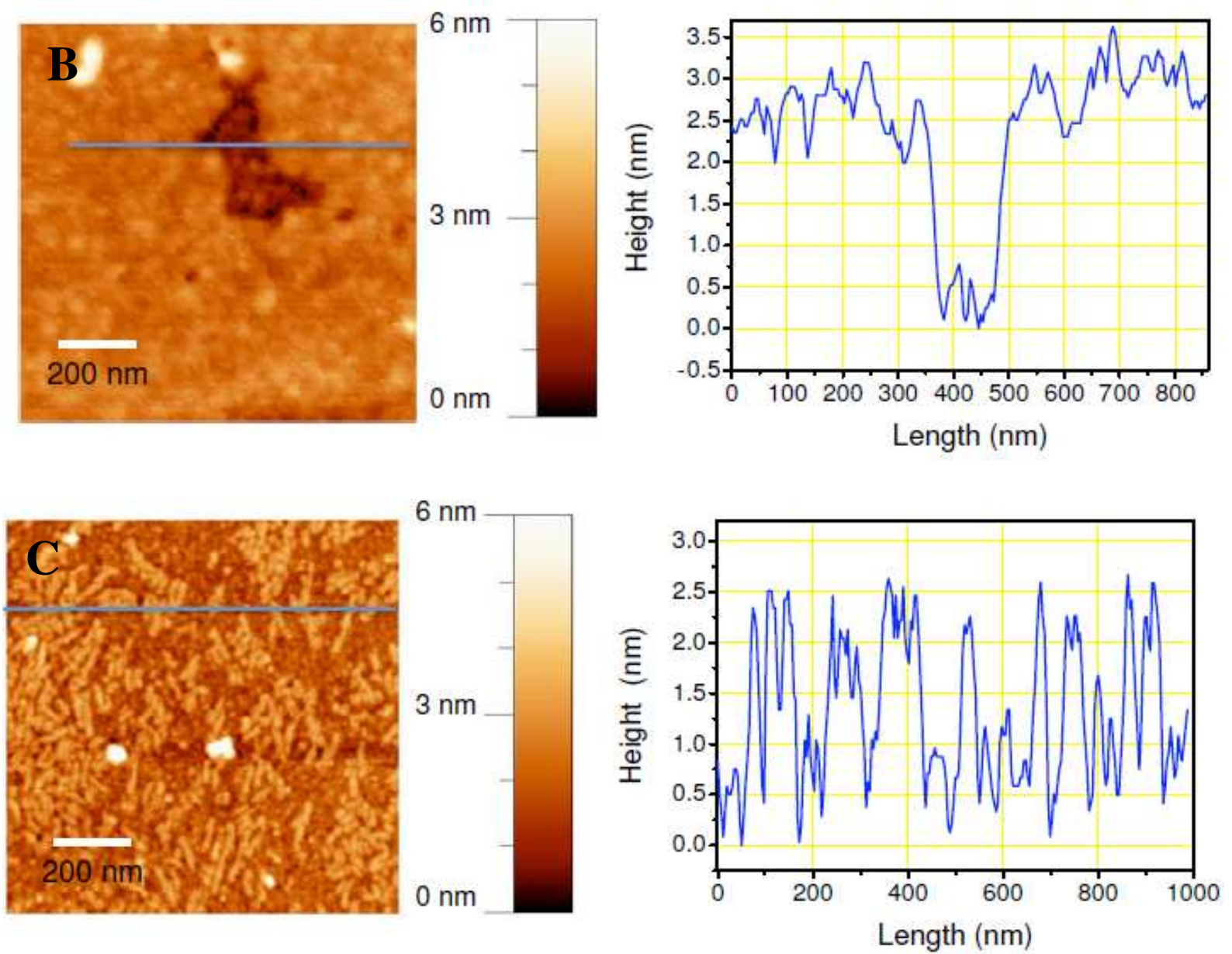

Figure 1. AFM topography images and height profiles of : (A), Vmh2 LB monolayer, (B) LS monolayer, (C) coexistence of LB monolayer and rodlets.

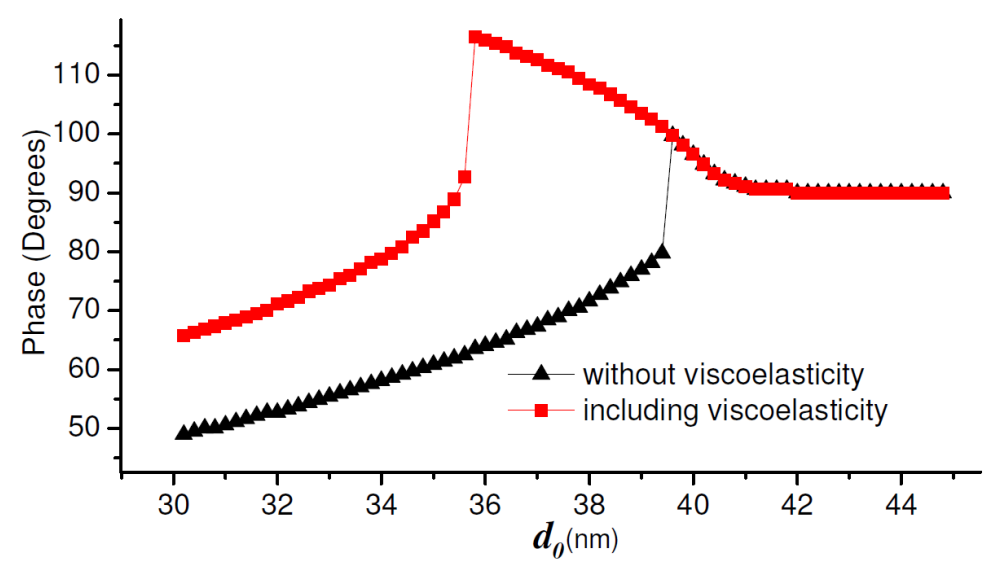

Figure 2. Simulated phase-distance curves without taking into account visco-elasticity (black color, triangles) and taking into account visco-elasticity (red color, squares). Parameters for the simulations are $: \omega_{0} / 2 \pi=142 \mathrm{kHz}, \mathrm{A}_{\mathrm{o}}=40 \mathrm{~nm}, \mathrm{k}_{\mathrm{c}}=5.1 \mathrm{~N} / \mathrm{m}, \mathrm{Q}=550, \mathrm{Q}_{\mathrm{dis}}=0.5, \mathrm{E}=65 \mathrm{GPa}, \mathrm{a}_{\mathrm{o}}=0.1 \mathrm{~nm}, \mathrm{H}=6 \times 10^{-20} \mathrm{~J}, \mathrm{R}=$ $10 \mathrm{~nm}$. 

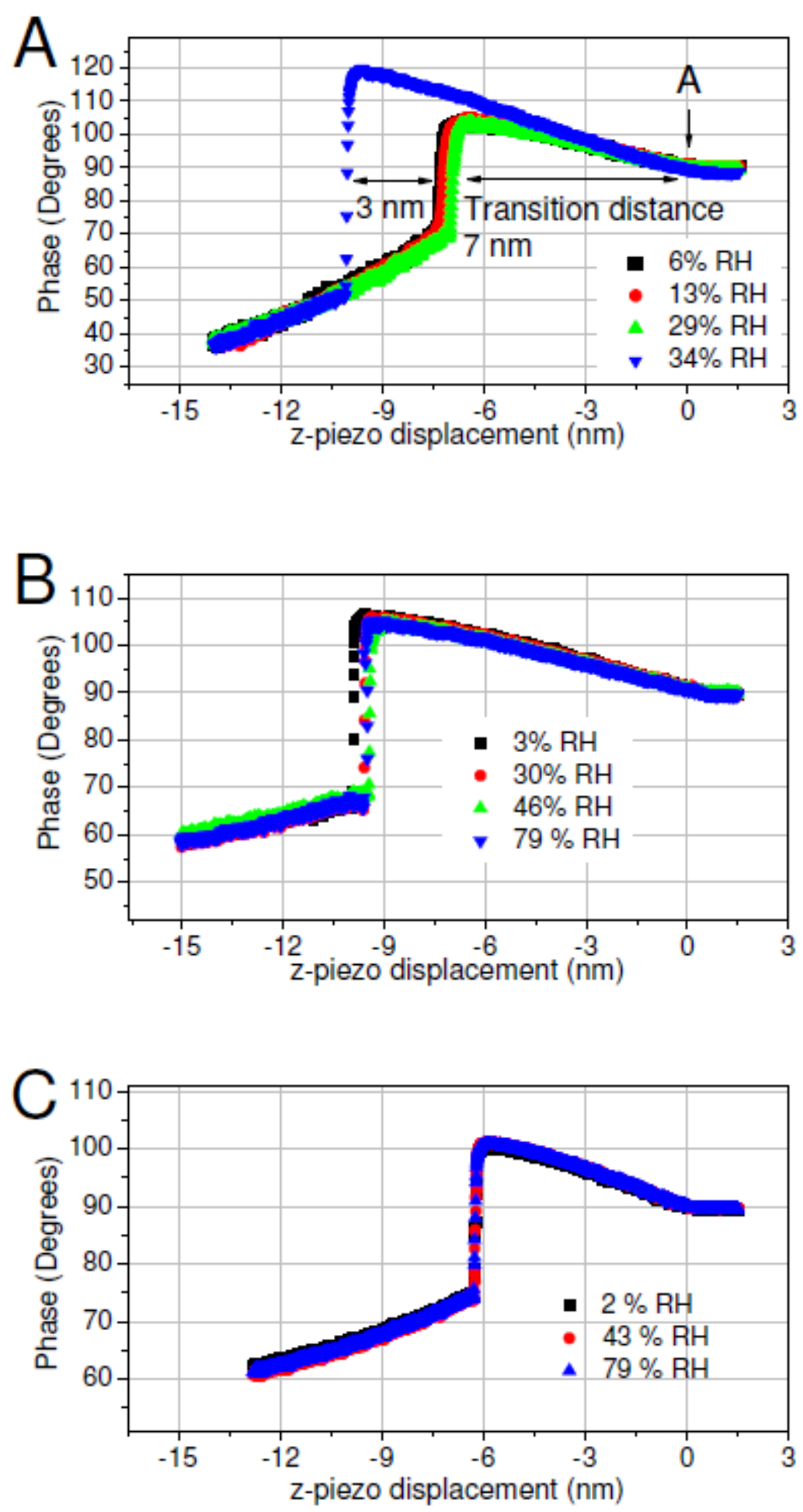

Figure 3. (A) Phase distance curves performed for Vmh2 LS film at different RH. " $A$ " indicates the onset of tip/sample interactions. (B) Phase distance curves performed for Vmh2 LB film at different humidities. (C) Phase distance curves for the rodlets at different humidities. For clarity purpose, phase signals measured at 10, 15, 20, 40, 50, 60 and $65 \% \mathrm{RH}$ are not shown in the graphs (B) and (C). 

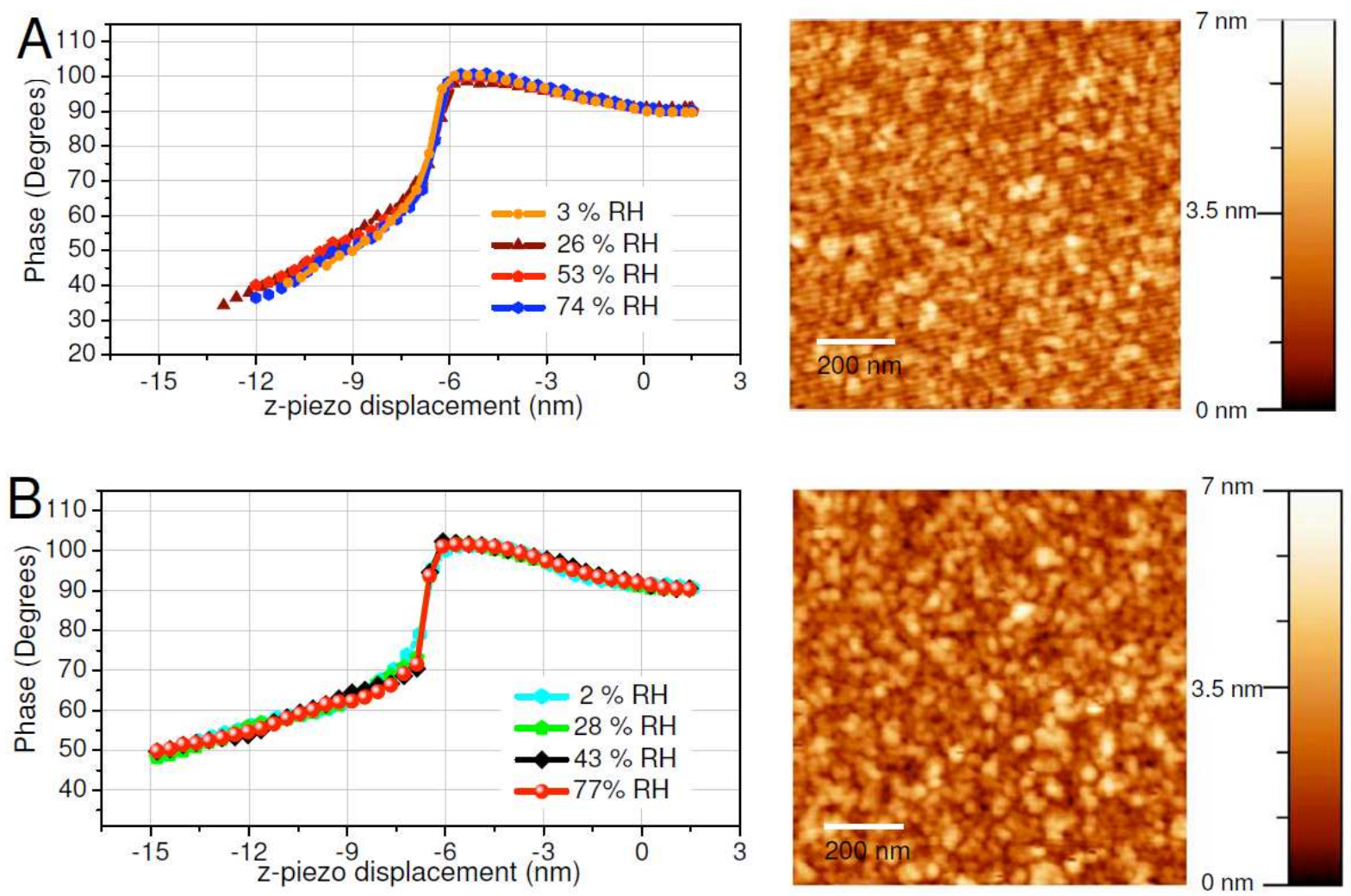

Figure 4. AFM topography images of the Vmh2 bilayers with the corresponding phase-distance curves for different humidities : (A) LB+LS bilayer deposited onto $\mathrm{SiO}_{2}$ surface. (B) LS+LB bilayer deposited onto silanized $\mathrm{SiO}_{2}$ surface.

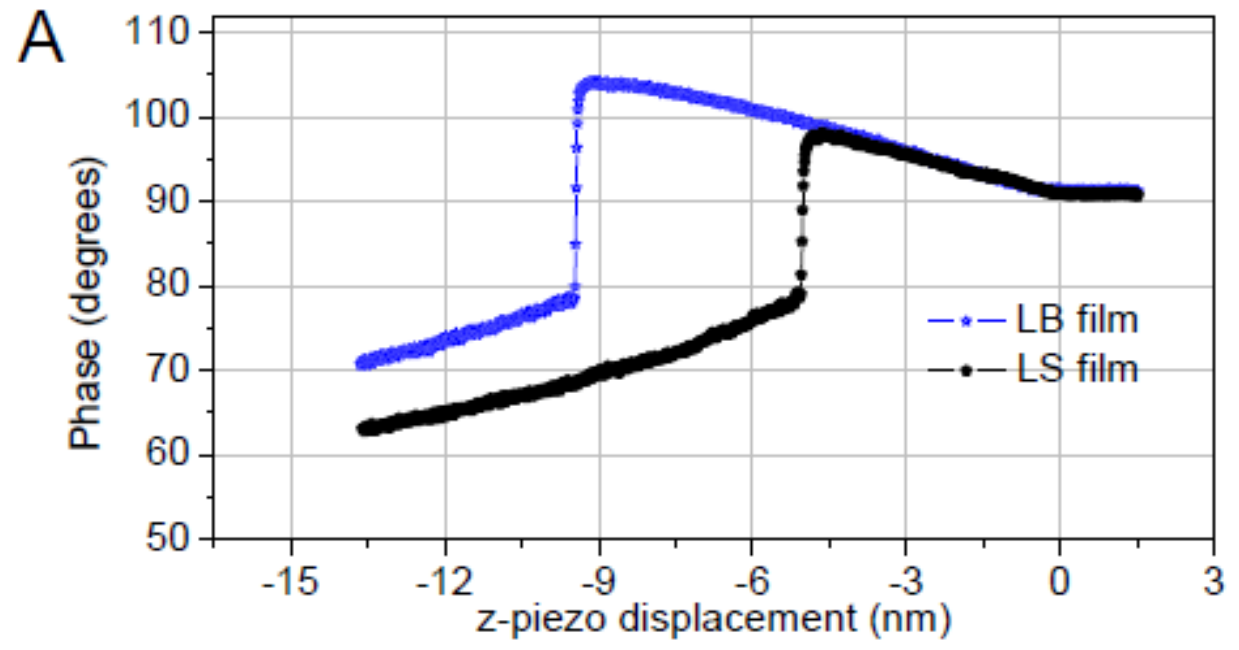




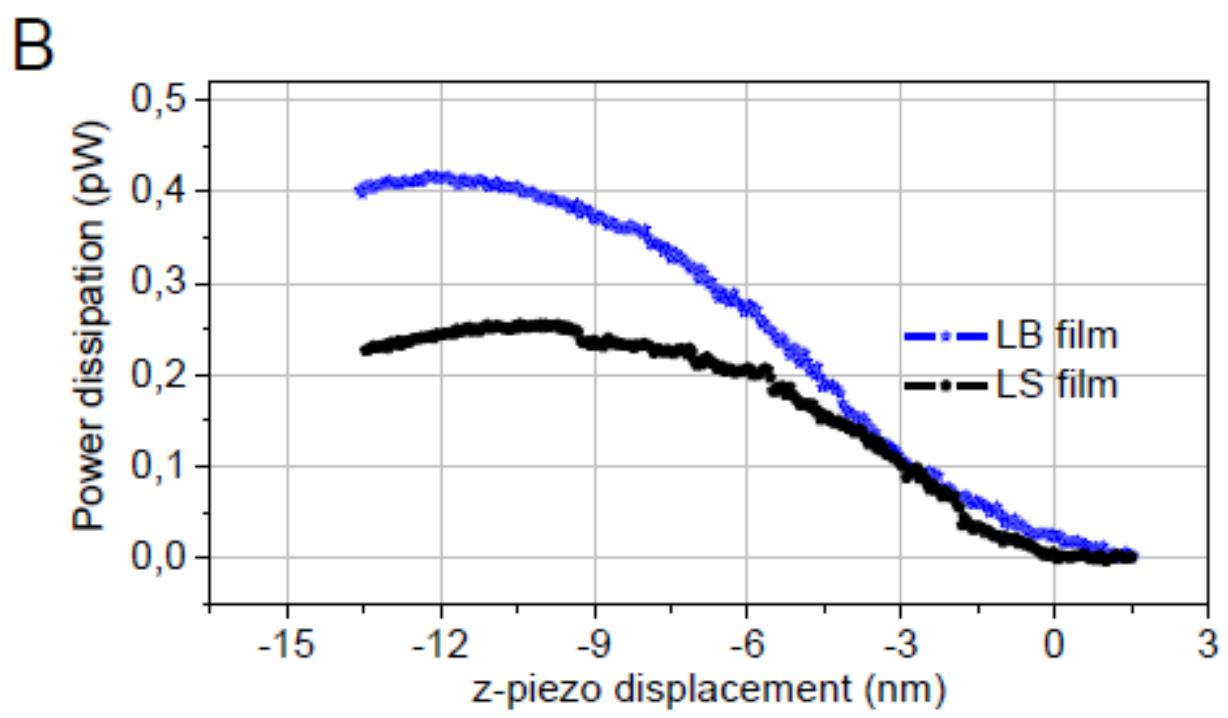

Figure 5. Experimental phase distance curve performed with the same tip for Vmh2 LB and LS monolayers in dry conditions (A), and the corresponding energy dissipation per period (B). The maximum dissipation observed for LB film $(0.42 \mathrm{pW})$ is about 1.7 times larger than the one observed for LS film ( $0.25 \mathrm{pW})$. The energy dissipation was evaluated using equation 3 .

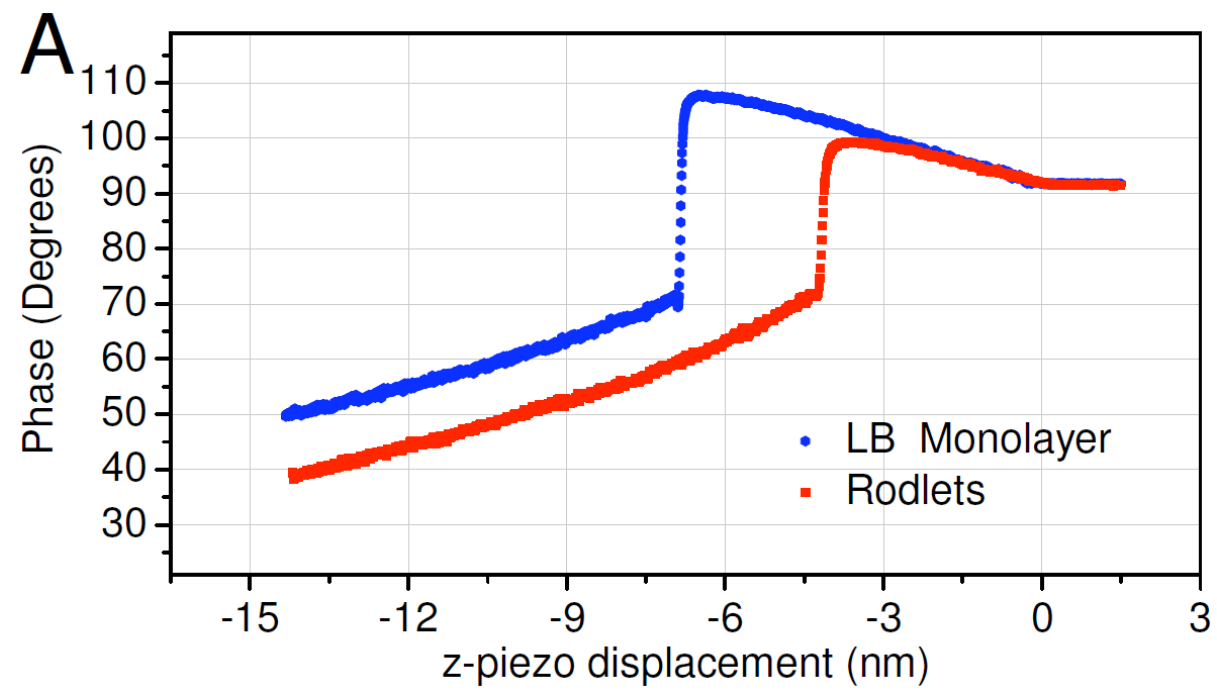




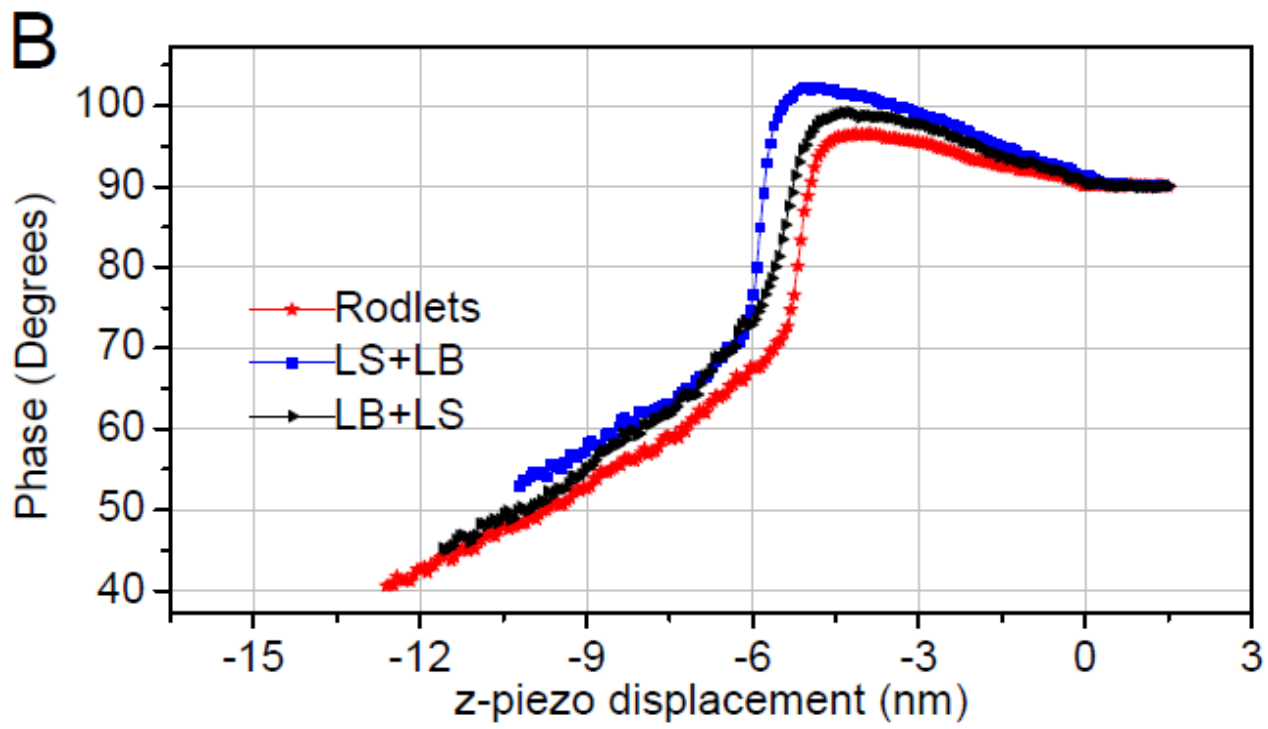

Figure 6. (A) Experimental phase distance curves performed in dry conditions with the same tip for LB monolayer and rodlets. (B) Experimental phase distance curves for hydrophobin bilayers and rodlets performed in dry conditions with the same tip.

\section{References}

(1) Linder, M. B.; Szilvay, G. R.; Nakari-Setälä, T.; Penttilä, M. E. FEMS Microbiol. ReV. 2005, 29, 877-896.

(2) Wosten, H. A. B.; de Vocht M. L. Biochim. et Biophys. Acta 2000, 1469, 79-86.

(3) Linder, M. B. Current Opinion in Coll. Interf. Science 2009, 14, 356-363.

(4) De Stefano, L.; Rea, I.; Giardina, P.; Armenante, A.; Rendina, I. Adv. Mat. 2008, 20, 1529-1533.

(5) Qin, M.; Wang, L. K.; Feng. X. Z. Langmuir 2007, 23, 4465-4471.

(6) Scholtmeijer, K.; Janssen, M. I.; Gerssen, B.; de Vocht, M. L.; van Leeuwen, B. M.; van Kooten, T.

G.;Wosten, H. A.; Wessels, J. G. Appl. EnViron. Microbiol. 2002, 68, 1367-1373.

(7) Wang, R.; Yang, Y. L.; Qin, M.; Wang, L. K.; Yu, L.; Shao, B.; Qiao, M. Q.; Wang, C.; Feng, X. Z. Chem. Mater 2007, 19, 3227-3231.

(8) Wang, Z.; Huang, Y.; Li, S.; Xu, H.; Linder, M. B. ; Qiao, M. Biosens. Bioelectr. 2010, 26, 1074-1079.

(9) Wang, Z.; Lienemannn, M.; Qiau, M.; Linder, M. B. Langmuir 2010, 26, 8491-8496. 
(10) Basheva, E. S.; Kralchevsky, P. A.; Danov, K. D.; Stoyanov, S. D.; Blijdenstein, T. B. J.; Pelan, E. G.; Lips, A. Langmuir 2011, 27, 4481-4488

(11) Janssen, M. I.; van Leeuwen, M. B.; Scholtmeijer, K.; van Kooten, T. G.; Dijkhuizen, L.; Wosten, H. A. Biomaterials 2002, 23, 4847-4854.

(12) Wessels J. Annual Review of Phytopathology 1994, 32, 413-437.

(13) Kwan, A. H. Y.; Winefield, R. D.; Sunde, M.; Matthews, J. M.; Haverkamp, R. G.; Templeton, M. D.; Mackay, J. P. Proceedings of the National Academy of Sciences of the United States of America 2006, 103, 362-3626.

(14) Yang, K.; Deng, Y.; Zhang, C.; Elasri, M. BMC bioinformatics 2006, 7(Suppl 4) S16.

(15) de Vocht, M. L.; Reviakine, I.; Ulrich, W. P.; Bergsma-Schutter, W.; Wosten, H. A. B.; Vogel, H.; Brisson, A.; Wessels, J. G. H.; Robillard, G. T. Protein Sci. 2002, 11, 1199-1205

(16) Askolin, S. Characterization of the Trichoderma reesei hydrophobins HFBI and HFBII. PhD Thesis (Helsinki University of Technology, Espoo) 2006, VTT Publications 601, ISSN 1235-0621.

(17) Kwan AH, et al. Macindoe, I.;Vukašin, P. V.; Morris, V. K.; Kass, I.; Gupte, R.; Mark, A. E.; Templeton, M. D.; Mackay, J. P.; Sunde, M. Journal of molecular biology 2008, 382, 708-720.

(18) Houmadi, S.; Ciuchi, F.; De Santo, M. P.; De Stefano, L.; Rea, I.; Giardina, P.; Armenante, A.; Lacaze, E.; Giocondo, M. Langmuir 2008, 24, 12953-12957.

(19) Armenante, A. ; Longobardi, S.; Rea, I.; De Stefano, L; Giocondo, M.; Silipo, A.; Molinaro, A.; Giardina, P. Glycobiology 2010, 20, 594-602.

(20) Malham, I. B.; Bureau L. Langmuir 2009, 25, 5631-5636.

(21) Yu, L.; Zhang, B.; Szilvay, G. R.; Sun, R.; Jänis, J.; Wang, Z.; Feng, S.; Xu, H.; Linder, M. B.; Qiao, M. Microbiology 2008, 154, 1677-1685.

(22) Butcher, J. C. Wiley and Sons, Ltd., Chichester, West Sussex PO19 8SQ, England 2003.

(23) Garcia, R.; San Paulo, A. Physical Review B 1999, 60, 4961-4967.

(24) Cleveland, J.; Anczykowski, B.; Schmid, A.; Elings, V. Applied Physics Letters 72, 2613. 
(25) Paajanen, M.; Katainen, J.; Pakarinen, O. H.; Foster, A. S.; Lahtinen, J. Journal of colloid and interface science 2006, 304, 518-523.

(26) van Honschoten, J.; Tas, N.; Elwenspoek, M. American Journal of Physics 2010, 78, 277- 286.

(27) Zitzler, L.; Herminghaus, S.; Mugele, F. Physical Review B 2002, 66, 155436.

(28) Wang, X.; Permentier, H. P.; Rink, R.; Kruijtzer, J. A.; Liskamp, R. M.; Wösten, H. A.; Poolman, B.; Robillard, G. T. Biophysical journal 2004, 87, 1919-1928.

(29) Kisko, K.; Torkkeli, M.; Vuorimaa, E.; Lemmetyinen, H.; Seeck, O. H.; Linder, M.; Serimaa, R. Surf. Sci. 2005, 584, 35-40.

(30) Wang, X.; De Vocht, M. L.; De Jonge, J.; Poolman, B.; Robillard, G. T. Protein Science 2002, 11, 1172-1181.

(31) Scholtmeijer, K.; de Vocht, M. L.; Rink, R.; Robillard, G. T.; Wosten, H. A. B. Journal of Biological Chemistry 2009, 284, 26309-26314 